\title{
THE RELATIONSHIP BETWEEN MARKERS OF MALNUTRITION AND MUSCLE WASTING WITH FRAILTY AND PHYSICAL FUNCTION IN OLDER CARE HOME RESIDENTS
}

\author{
A. Slee', T. Ahmed², L. Storey², L. Wilkinson², G. Wilson², G. Garden ${ }^{3}$
}

\begin{abstract}
Background: Older care homes residents may suffer from malnutrition and muscle wasting within a background of varying degrees of frailty, comorbidity and disability. Hence, malnutrition is complicated by co-presence of sarcopenia, cachexia and inactivity-induced muscle atrophy. Objectives: (1) to assess the prevalence of malnutrition in care home residents using different methodologies. (2) To examine the relationship between measurements of nutritional status and muscle mass with frailty and physical function; Design: initial pilot study. Setting: care homes for older people. Participants: 73 participants, 46 female and 27 male; Intervention: observational study. Measurements: height (m), weight (kg), body mass index (BMI) (kg), bioelectrical impedance assessment (BIA) of fat free mass index (FFMI) (kg/m2), mid upper arm muscle circumference (MUAMC) (cm), Edmonton Frailty Scale (EFS) and Barthel Index (BI). Results: There was a relatively high prevalence of malnutrition depending on measure used. MNA-SF 0-7 score was 30\% for females and 28\% males. Low MUAMC was found in $41 \%$ females and 53\% males; low BIA FFMI in 37\% females and 52\% males. Good correlation $(\mathrm{P}<0.001)$ was found for most measures including against EFS and BI for MNA-SF and MUAMC. Conclusions: Malnutrition prevalence was relatively high. MNA-SF and MUAMC correlated well with functional status and frailty EFS measures. FFMI by BIA correlated well with MNA-SF and MUAMC. This range of practical techniques should be explored further for determining malnutrition risk and muscle wasting in relation to functionality and frailty in care home residents
\end{abstract}

Key words: Malnutrition, sarcopenia, cachexia, frailty, muscle wasting.

\section{Introduction}

Older people in care homes have varying degrees of comorbidity, frailty and impaired functional ability which may be associated with clinical outcomes (1). Malnutrition, a serious concern for this population group, is a component of the frailty cycle, and may be linked to worse outcomes $(2,3)$, therefore screening for malnutrition with simple tools has high clinical value $(4$, $5)$. There is debate however, regarding which methods to use and specific cut-off points (e.g. body mass index, BMI) (6). Furthermore, differentiating the different states of cachexia, sarcopenia and disuse atrophy is complex (7-10). Older people with varying degrees of frailty, comorbidity (and associated inflammation etc.) and poor physical function may suffer from a combination of states and be difficult to assess. Regardless of origin, these states

1. Institute for Liver and Digestive Health, UCL Medical School, Royal Free Campus, Rowland Hill Street, Hampstead, London, NW3 2PF; 2. United Lincolnshire Hospitals NHS Trust, Lincoln County Hospital, Greetwell Road Lincoln, LN2 5QY; 3. St Barnabas Hospice, 36 Nettleham Road, Lincoln, LN2 1RE

Corresponding Author: Dr Adrian Slee, Institute for Liver and Digestive Health, UCL Medical School, Royal Free Campus, Rowland Hill Street, Hampstead, London, NW3 2PF, United Kingdom, email: adrianslee@hotmail.co.uk. lead to skeletal muscle mass (SMM) loss and a reduction in nutritional status making an older person more susceptible to malnutrition and risk of morbidity and mortality. Recently, the term 'muscle wasting disease' has been suggested as an umbrella term to encompass all forms of muscle loss (11).

Regarding techniques of assessment for malnutrition risk, the mini nutritional assessment (MNA) and abbreviated short-form (MNA-SF) have been validated and suggested for use in older people (4) and uses BMI with significant weight loss and other specific questions. Previous studies in older people have also utilised bioelectrical impedance assessment (BIA) to estimate nutritional status, measuring fat free mass (FFM) and FFM index (FFMI in $\mathrm{kg} / \mathrm{m} 2)(5,12-14)$. Recently, an ESPEN consensus statement, produced for the assessment of malnutrition discussed specific cut-off points for BMI, weight loss and use of FFMI. Muscle wasting can be estimated by FFMI as an indicator of SMM. It can also be measured practically by the mid upper arm muscle circumference (MUAMC). The MUAMC was used in a large Italian study $(n=357)$ by Landi et al (IISIRENTE Study) which investigated the relationship between MUAMC in community-dwelling older people with 
physical performance and mortality (15).

One area of research has been the development of tools and measures for frailty status and the relationship with health and specific aspects such as muscle loss in ageing, sarcopenia. The Edmonton Frailty Scale (EFS) was developed as a brief, valid and reliable tool which can be used to identify multi-domain frailty by clinical staff without training in geriatric medicine (16). The usefulness of the EFS in care homes and relationship with markers of nutritional status, malnutrition and muscle wasting has yet to be ascertained. Furthermore, the relationship between these different markers with measurements of the Activities of Daily Living (ADLs) is also of high interest. The Barthel Index (BI) is commonly used by geriatricians to indicate functional ability/disability (17).

This study aimed to (1) investigate malnutrition prevalence in care home residents using different methods and (2) investigate the relationship between markers of nutritional status, frailty and physical function.

\section{Methods}

\section{Participants and study design}

This study was undertaken between October 2015 and May 2016 and is part of an ongoing care home service evaluation, the Frailty and Nutrition Study in Lincoln (FANS). Study was cleared through NHS research ethics committee in September 2015. Care home residents underwent Comprehensive Geriatric Assessment (CGA) in four care homes in Lincoln, United Kingdom. Patients were diagnosed with different levels of frailty and with a range of comorbidities including; cardiovascular disease, chronic heart failure, chronic kidney disease, chronic obstructive pulmonary disorder, cancer, diabetes, arthritis, and dementia. Most residents were being treated with multiple drugs. The aim was to recruit 100 to 150 patients in line with other similar studies; however the designated study time restraints dictated the current number. Measurements were collected by members of a multidisciplinary care team.

\section{Anthropometric measurements}

Height (in $\mathrm{m}$ ) was estimated using ulnar length and conversion tables (BAPEN, UK). Weight (in $\mathrm{kg}$ ) was measured and body mass index (BMI in $\mathrm{kg} / \mathrm{m} 2$ ) calculated. Mid upper arm circumference (MUAC) was measured using a tape measure around the mid-point of the upper arm. Measurements were taken on the right side of the participant's body unless affected by disability or disease.

\section{Bioelectrical impedance assessment measurements}

BIA measurements were taken using a singlefrequency $(50 \mathrm{kHz})$ Maltron $916 \mathrm{~S}$, bioelectrical impedance analyser (Maltron International Ltd., Rayleigh, Essex, UK). Measurements were taken using a standard hand-to-foot tetra-polar technique with participants in the supine position, in accordance with the manufacturer's guidelines. Raw impedance measurements of resistance $(\mathrm{R})$ and reactance $\left(\mathrm{X}_{\mathrm{c}}\right)$ in ohms and PA were recorded.

The BIA estimation of FFM was completed using the following BIA equation (Kyle equation (18)):

$\mathrm{FFM}=-4.1041(0.518 \times$ height $2 / \mathrm{R})+(0.231 \times$ weight $)+$ $\left(0.130 \times X_{C}\right)+(4.229 \times$ sex: men $=1$, women $=0)$. Height is in $\mathrm{cm}$ and weight in $\mathrm{kg}$.

\section{Nutritional assessment: MNA-SF screening}

MNA-SF screening was undertaken by clinical staff according to instructions and scores recorded. Scores were converted into categories for nutritional status using MNA scoring criteria either low risk/normal (12-14), medium risk/at risk (8-11) and high risk/malnourished (0-7).

\section{Mid-upper arm muscle circumference calculation}

The MUAMC was calculated using the formula:

MUAMC $=$ mid-upper arm circumference $-(3.14 \mathrm{X}$ triceps skinfold thickness)

Measurement of triceps skinfold thickness (to the nearest $0.2 \mathrm{~mm}$ ) was made using Harpenden skinfold calliper (range: $0.00-50.00 \mathrm{~mm}$; minimum graduation: $0.20 \mathrm{~mm}$ ).

Using reference data from Landi et al, the lowest tertiles for males $(<21.1 \mathrm{~cm})$ and for females $(<19.2 \mathrm{~cm})$ were used as cut-off points to indicate low muscle mass.

\section{Malnutrition prevalence}

Prevalence of malnutrition was assessed by BMI, MNA-SF score and FFMI. A BMI of $<20 \mathrm{~kg} / \mathrm{m} 2$ was used as the population is older and high presence of comorbid chronic conditions. E.g. in the cachexia definition by Evans et al. a BMI $<20 \mathrm{~kg} / \mathrm{m} 2$ is used as a cut-off point when there is presence of a chronic disease (7).

\section{Edmonton frailty scale}

The EFS was undertaken by clinical staff as part of routine CGA in participants. The EFS 10 domain test as described by Rolfson et al with maximum score of 17 was undertaken (16). Higher scoring indicates increasing frailty. 


\section{Barthel index}

The BI was undertaken by clinical staff as part of routine CGA in participants. A standard 10 question BI with a maximum of 20 point scoring was undertaken (17). Lower scoring indicates increasing disability.

\section{Statistical analysis}

Data was analysed as a group and individually for males and females. Cut-off points were assigned for malnutrition risk and low MUAMC. Number of residents and percentage $(\%)$ was calculated for prevalence. Correlations were performed on all variables using Pearson test and Spearman for nonparametric data. All statistical tests were performed using IBM SPSS Statistics Version 21.

\section{Results}

There were 73 resident participants recruited over 4 separate care homes. The characteristics of the older care home residents can be seen in Table 1. MNA-SF and BIA was completed in all residents, MUAMC in 58, EFS in 49 and $\mathrm{BI}$ in 52.

\section{Table 1}

Participant characteristics and variables. Mean +/- standard deviation, median [ ], minimum and maximum ( )

\begin{tabular}{ll}
\hline Number & 73 (46 females, 27 males) \\
Age, years & $86.0+/-6.5$ years of age [87] (70-97) \\
Height, $\mathrm{m}$ & $1.65+/-0.09 \mathrm{~m}$ [1.65] (1.47-1.91) \\
Weight, kg & $66.1+/-16.9 \mathrm{~kg}[63.5](38.7-117.7)$ \\
Body mass index (BMI), kg/m2 & $24.3+/-6.2 \mathrm{~kg} / \mathrm{m} 2[23.0](15.3-49.0)$ \\
Mini nutritional assessment (MNA) score & \\
$\begin{array}{l}\text { (0-14) } \\
\text { Edmonton frail scale (EFS) (0-17) }\end{array}$ & $9.0+/-3.0[9.5](3-14)$ \\
Barthel index (0-20) & $11.2+/-2.8[11](5-16)$ \\
Fat Free Mass Index, kg/m2 & $10.3+/-6.6[11](0-20)$ \\
$\begin{array}{l}\text { Mid-upper arm muscle circumference } \\
\text { (MUAMC), cm }\end{array}$ & $16.2+/-2.5[16.0](11.4-24.2)$ \\
\hline
\end{tabular}

All residents had gait speed and grip strength below the cut-off points for the European Working Group on Sarcopenia in Older Persons (EWGSOP) definition for sarcopenia (9).

Prevalence of malnutrition was assessed by BMI and MNA-SF score (see Table 2). Prevalence of low MUAMC indicative of muscle wasting can be found in Table 2 along with low FFMI.

\section{Correlations}

There was good correlation between most measures (Table 3). However, there was no significant correlation between FFMI with Edmonton EFS or Barthel Index BI. Figures 1-3 depicts key correlations for (1) MNA-SF score, (2) MUAMC and (3) FFMI.

Table 2

Prevalence of malnutrition, low MUAMC and low FFMI

\begin{tabular}{lll}
\hline & Females & Males \\
\hline Malnutrition $<20 \mathrm{~kg} / \mathrm{m} 2$ & $11 / 46(24 \%)$ & $6 / 27(22 \%)$ \\
Malnutrition 0-7 MNA-SF & $13 / 43(30 \%)$ & $7 / 25(28 \%)$ \\
Malnutrition risk 8-11 MNA-SF & $15 / 43(35 \%)$ & $12 / 25(48 \%)$ \\
Low MUAMC* & $14 / 34(41 \%)$ & $10 / 19(53 \%)$ \\
Low FFMI $\dagger$ & $17 / 46(37 \%)$ & $13 / 25(52 \%)$ \\
\hline${ }^{*}$ Low MUAMC: $<19.2 \mathrm{~cm}$ for females and $<21.1 \mathrm{~cm}$ for males. +Low FFMI: $<15$ \\
$\mathrm{~kg} / \mathrm{m} 2$ for females and $<17 \mathrm{~kg} / \mathrm{m} 2$ males.
\end{tabular}

Table 3

Correlations between variables with correlation coefficient, $r$ and significance, $P$ values shown

\begin{tabular}{lll}
\hline Variables & $\begin{array}{l}\text { Correlation } \\
\text { coefficient, r value }\end{array}$ & Significance, P value \\
\hline MNA-SF vs BMI & 0.68 & $<0.001$ \\
MNA-SF vs EFS & -0.75 & $<0.001$ \\
MNA-SF vs BI & 0.58 & $<0.001$ \\
MUAMC vs BMI & 0.64 & $<0.001$ \\
MUAMC vs MNA-SF & 0.49 & $<0.001$ \\
MUAMC vs EFS & -0.61 & $<0.001$ \\
MUAMC vs BI & 0.43 & 0.01 \\
FFMI vs BMI & 0.72 & $<0.001$ \\
FFMI vs MUAMC & 0.51 & $<0.001$ \\
FFMI vs MNA-SF & 0.43 & $<0.001$ \\
BMI vs EFS & -0.53 & $<0.001$ \\
BMI vs BI & 0.27 & 0.063 \\
EFS vs BI & -0.71 & $<0.001$ \\
\hline
\end{tabular}

\section{Discussion}

In this study, 73 care home residents were screened for malnutrition using BMI, the MNA-SF and BIA estimation of FFMI (Table 2). Malnutrition by BMI was $24 \%$ in females and $22 \%$ in males. A BMI cut-off point of $20 \mathrm{~kg} /$ $\mathrm{m} 2$ was used to indicate malnutrition rather than $18.5 \mathrm{~kg} /$ $\mathrm{m} 2$. This was due to the age of the participants $(86+/-6.5$ years) with similar studies using a higher cut-off point for older people, as does the MNA-SF tool. Furthermore, the cachexia definition by Evans et al, utilises a cut-off point of $20 \mathrm{~kg} / \mathrm{m} 2$ in the presence of a chronic condition, 
e.g. heart failure or cancer etc. (7). The population group that were assessed in this study had a high prevalence of comorbidity and chronic disease conditions. An ESPEN consensus paper recently suggested using $18.5 \mathrm{~kg} / \mathrm{m} 2$ OR significant unintentional weight loss (>10\% indefinite of time, or $>5 \%$ over the last 3 months) combined with either BMI $(<20 \mathrm{~kg} / \mathrm{m} 2$ if $<70$ years of age, or $<22 \mathrm{~kg} / \mathrm{m} 2$ if $\geq 70$ years of age $)$ or FFMI $(<15 \mathrm{~kg} / \mathrm{m} 2$ and $17 \mathrm{~kg} / \mathrm{m} 2$ in women and men, respectively) (6). In this participant group, weight loss was difficult to assess accurately as it was highly dependent on robust records being kept within the care home itself (e.g. previous carers etc.). Weight loss is a component of frailty, a strong predictor of outcomes and in particular, the presence of cachexia wasting. As described above, due to high comorbidity and chronic disease prevalence it is likely that cachexia prevalence was relatively high despite not having the weight loss data to confirm this.

\section{Figure 1}

Graphs to show the relationship between MNA-SF score and (a) BMI, (b) EFS and (c) BI. Closed circles indicates female residents and triangles, males. Correlation results can be found within Table 3

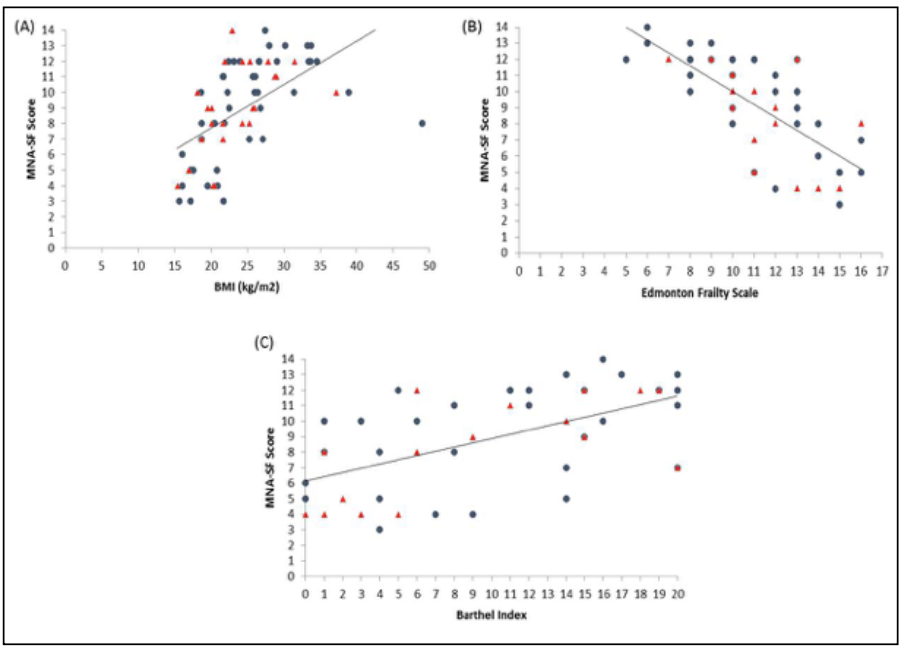

Identifying malnutrition by MNA-SF found that $30 \%$ females and $28 \%$ males were classified as malnourished (0-7 score) and $35 \%$ females and $48 \%$ males as 'at risk' (score 8-11). This was a higher prevalence than using BMI. Based upon the nature of the MNA-SF and the questions it contains, it may be suggested that a person who has greater frailty, comorbidity and physical disability will score worse with a greater risk of malnutrition. Interestingly, the correlation results tend to confirm this relationship with BMI $(\mathrm{r}=0.68, \mathrm{P}<0.001)$, EFS $(\mathrm{r}=-0.75$, $\mathrm{P}<0.001)$ and $\mathrm{BI}(\mathrm{r}=0.58, \mathrm{P}<0.001)$ (Figure 1$)$.

\section{Figure 2}

Graphs to show the relationship between MUAMC and (a) BMI, (b) MNA-SF score and (c) EFS and (d) BI. Closed circles indicates female residents and triangles, males.

Correlation results can be found within Table 3

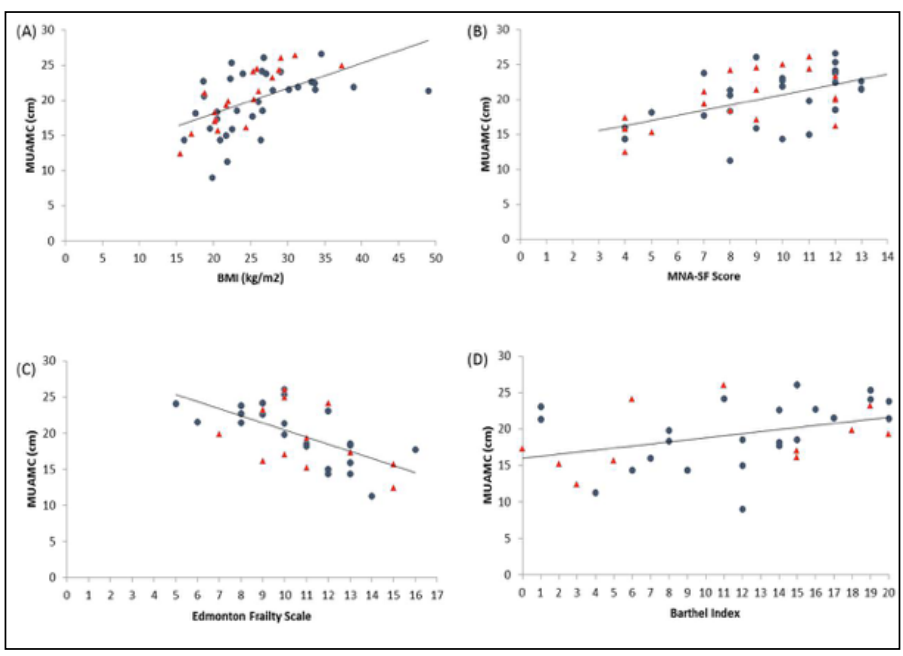

Figure 3

Graphs to show the relationship between FFMI and (a) BMI, (b) MUAMC and (c) MNA-SF score. Closed circles indicates female residents and triangles, males.

Correlation results can be found within Table 3

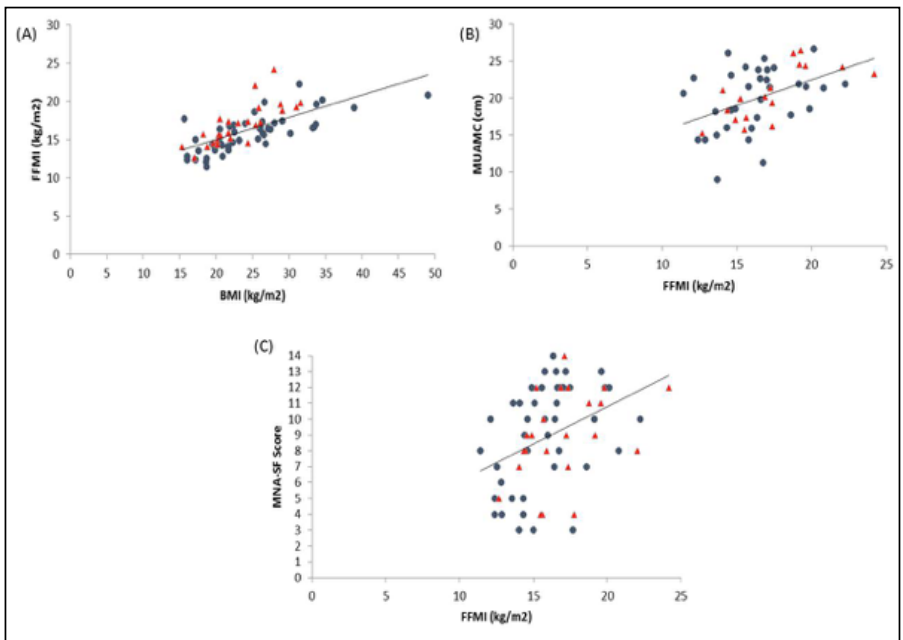

BIA estimation of FFMI identified that $37 \%$ females and $52 \%$ males had a low FFMI. Cut-off points of $<15 \mathrm{~kg} /$ $\mathrm{m} 2$ for females and $<17 \mathrm{~kg} / \mathrm{m} 2$ males were utilised as suggested from the ESPEN consensus paper to indicate a low FFMI (6). BIA estimations of FFMI prove to be useful in this study similar to previous work (5). In the previous study, BIA estimation of FFMI was used alongside MNA-SF to better categorise malnutrition risk compared to using the standard UK Malnutrition Universal Screening Tool (MUST). With regards to accuracy of BIA, dual energy x-ray absorptiometry is the gold standard technique for measuring FFMI, but is difficult to use in the older population group in long term care and is also expensive. BIA is inexpensive and 
portable and can be used at the bed side (e.g. bed-bound residents). Drawbacks to its use however, include errors due to hydration abnormalities leading to false FFM estimations. In this study, 2 residents were omitted from FFMI estimation due to hydration abnormalities. Also, the presence of an electronic cardiac pacemaker is contraindicated for BIA use, and which is more likely to be prevalent in this population. Within the ESPEN consensus paper it was suggested that FFMI should be used as a possible measure of nutritional status, alongside weight loss (6). It may also be suggested however that FFMI alone may be useful in situations when it is impossible to gather accurate weight loss information.

In terms of skeletal muscle mass (SMM) the FFMI is a useful predictor of both nutritional status and an indicator of overall SMM. FFM consists of all mass other than fat mass and obviously the large body compartment of SMM makes up a high proportion of FFM. Therefore, we may assume under normal circumstances that a low FFM and FFMI may be indicative of a poor nutritional status and also low SMM. The FFMI was positively correlated with BMI $(\mathrm{r}=0.72, \mathrm{P}<0.001)$, MNA-SF ( $\mathrm{r}$ $=0.43, \mathrm{P}<0.001)$ and MUAMC $(\mathrm{r}=0.51, \mathrm{P}<0.001)$ in residents. MUAMC was measured as a practical means of estimating SMM and muscle wasting. Using the lower tertiles in a study by Landi et al as cut-off points, the relative muscle mass and number of people with a lower MUAMC was determined. The number of participants with a low MUAMC was $41 \%$ females and $53 \%$ males. In particular, interestingly the prevalence rates were quite similar to the low FFMI levels (37\% females and $52 \%$ males). Hence, this data may support the concept of FFMI as an estimation of SMM. Landi et al found in community-dwelling older people that those with a higher MUAMC tertile had better physical performance (measured using a $4 \mathrm{~m}$ walk speed test, Short Physical Performance Battery score and hand grip strength) and a lower risk of death (adj. hazard ratio $0.45 ; 95 \%$ confidence interval 0.23-0.87). In our study, residents either had a low walking speed and hand grip strength below EWGSOP sarcopenia cut-off points (data not shown here), or presence of disability. Interestingly, there was a significant correlation between MUAMC with EFS ( $\mathrm{r}=$ -0.61, $\mathrm{P}<0.001)$ and $\mathrm{BI}(\mathrm{r}=0.43, \mathrm{P}=0.01)$.

The reduced muscle mass may be the result of a combination of age-related sarcopenia, chronic disease, e.g. cachexia, and physical inactivity/disability (7-11). Practically however, this is difficult to untangle and the overarching term of muscle wasting disease which has recently been suggested by Von Haehling et al may be important here (11). Dupuy et al, found in a large cohort of older women $(n=3025)$ that sarcopenia prevalence can vary greatly depending on the method used (19). Hence, there needs to be a standardisation in the terminologies and methods used in measuring sarcopenia and overall muscle loss.

The EFS was measured in participants to assess frailty. The EFS is a simple tool which can be used by non- geriatrician staff to assess multi-domain frailty which includes sections on cognition, mood, medications and functional status. The mean score of the participants was $11.2+/-2.8$, which indicates a moderate level of frailty. As stated earlier, significant correlations were found between EFS and MNA-SF and with MUAMC. Furthermore, a significant negative correlation was found between BMI and EFS $(r=-0.53, P<0.001)$ (Table 3). This data fits well with the concept of the frailty cycle. Fried et al, describe this relationship in detail in (3). Nutritional status and malnutrition risk are key components of frailty due to the impact of a variety of factors including, the dysregulation of energy balance with ageing (anorexia of ageing) and illness (inflammation driven changes in appetite and metabolism). Furthermore, sarcopenia is also a major component. Interestingly, in this study there was no correlation with FFMI which was perhaps unexpected. This may be due to low study participant numbers. The EFS was evaluated in a recent study by Perna et al with 366 hospitalised older patients (20). EFS scores were associated with cognition, functional independence, medications, nutritional status by MNA, functional performance by BI and hand grip strength. They also found a significant difference in female patients with sarcopenia (SMM Index by DEXA). They concluded that the EFS may be a helpful tool for stratifying the state of fragility in this population group.

Frailty and sarcopenia increases the risk of disability $(1,3)$. In this study the BI was taken as a method of measuring ADLs and hence physical functional status. The mean score of the participants was $10.3+/$ 6.6 which may indicate a moderately impaired level of functional ability, but there was also a high level of variance between participants. EFS score highly correlated (negatively) with BI $(\mathrm{r}=-0.71, \mathrm{P}<0.001)$ (Table 3), such that increasing frailty was associated with worsening of physical function. As stated earlier, significant correlations were found between BI and MNA$\mathrm{SF}$ and with MUAMC. Furthermore, a significant positive correlation was found between BMI and BI $(r=0.27, \mathrm{P}<$ 0.063) (Table 3). This data would suggest that functional decline relates to poor nutritional status and muscle mass. Villafane et al found that BI was positively associated with MNA-SF score in 344 older rehabilitation centre patients (i.e. higher scoring indicating better functional status and nutritional status etc.) (21). In addition, similar results were found in a large Spanish study with 895 institutionalised older residents across 34 nursing homes, whereby MNA was positively associated with BI (22). Zuliani et al, performed a 2 year longitudinal nursing home study in Italy with 98 participants (23). They found that malnutrition predicted further worsening of functional status and that the decline in body cell mass (measured by BIA) was proportional to the loss in ADLs. Furthermore, Cereda et al specifically investigated the relationship between the MNA score and nutritional status with functional status by BI in 123 older people in long term care (24). MNA significantly correlated with BI 
$(\mathrm{r}=0.55, \mathrm{P}<0.0001)$ and a poorer functional status was associated with low BMI, low MUAMC and reduced oral intake. Hence, this data corroborates findings from our study.

This study suggests that multi-domain screening for nutritional status, muscle mass, frailty and functionality is important in this population group. Regular screening may improve diagnosis and guide treatment opportunities, e.g. nutritional and protein supplementation. Further studies are required to confirm this and to evaluate specific methods e.g. determination of malnutrition prevalence.

\section{Conclusion}

This pilot study found that prevalence of malnutrition was dependent on the method used to determine. There was a high prevalence of malnutrition by MNA-SF and FFMI and high levels of muscle wasting by MUAMC and FFMI. Those residents with poor nutritional status (by BMI, MNA-SF and FFMI) had lower muscle mass, greater frailty (by EFS) and worse physical function (by BI). Future studies should be performed to confirm or refute these relationships and their meaning.

Funding: The Bromhead Medical Charity, Lincoln, part-funded this study. The sponsors had no role in the design and conduct of the study; in the collection, analysis, and interpretation of data; in the preparation of the manuscript; or in the review or approval of the manuscript.

Conflict of interest: The authors declare that they have no conflicts of interest.

Statement of Authorship: AS is the lead author and designated study Chief Investigator. GG and TA played key roles in the design and development of the study and in writing of the paper. LS, LW and GW were co-investigators primarily involved in data acquisition.

Acknowledgements: We wish to thank all of the care home staff and residents and the Bromhead Medical Charity, Lincoln.

Ethical standards: Full UK NHS research ethics guidelines were followed in this study.

\section{References}

1. Fried LP, Ferrucci L, Darer J, Williamson JD, Anderson G. Untangling the concepts of disability, Frailty, and Comorbidity: Implications for improved targeting and care. The Journals of Gerontology Series A: Biological Sciences and Medical Sciences. 2004;59(3):M255-M263.

2. Norman K, Pichard C, Lochs H, Pirlich M. Prognostic impact of diseaserelated malnutrition. Clinical Nutrition. 2008;27(1):5-15. doi:10.1016/j clnu.2007.10.007.

3. Fried LP, Tangen CM, Walston J, Newman AB, Hirsch C, Gottdiener J, et al. Frailty in older adults: evidence for a phenotype. J Gerontol A Biol Sci Med Sci 2001;56:M146-56.
4. Rubenstein LZ, Harker JO, Salva A, Guigoz Y, Vellas B. Screening for undernutrition in geriatric practice: developing the short-form mini nutritional assessment (MNA-SF). J Geront 2001;56 A:M366-77.

5. Slee A, Birch D, Stokoe D. A comparison of the malnutrition screening tools, MUST, MNA and bioelectrical impedance assessment in frail older hospital patients. Clin Nutr. 2015; 34(2):296-301. doi: 10.1016/j.clnu.2014.04.013.

6. Cederholm T, Bosaeus I, Barazzoni R, Bauer J, Van Gossum A, Klek S et al. Diagnostic criteria for malnutrition - An ESPEN consensus statement. Clin Nutr 2015; 34:441-47.

7. Evans WJ, Morley JE, Argiles J, et al. Cachexia: A new definition. Clin Nutr. 2008; 27: 793-799.

8. Muscaritoli M, Anker SD, Argilés J, Aversa Z, Bauer JM, Biolo G et al Consensus definition of sarcopenia, cachexia and pre-cachexia: joint document elaborated by Special Interest Groups (SIG) "cachexia-anorexia in chronic wasting diseases" and "nutrition in geriatrics". Clin Nutr. 2010; 29(2): 154-9.

9. Cruz-Jentoft AJ, Baeyens JP, Bauer JM, Boirie Y, Cederholm T, Landi F et al. Sarcopenia: European consensus on definition and diagnosis: Report of the European Working Group on Sarcopenia in Older People. Age Ageing 2010; 39(4): 412-423.

10. Evans WJ. Skeletal muscle loss: Cachexia, sarcopenia, and inactivity. American Journal of Clinical Nutrition. 2010;91(4):1123S-1127S.

11. Von Haehling S, Morley JE, Coats AJS, Anker SD, Rosano G, Bernabei R, K. Kalantar-Zadeh K. Muscle wasting disease: a proposal for a new disease classification. J Cachexia Sarcopenia Muscle 2014; 5:1-3.

12. Slee A. Estimating nutritional status in a small cohort of elderly care home residents using MUST, MNA and bioelectrical impedance phase angle and vector analysis. J Aging Res Clin Practice 2013;2(1):65-70.

13. Slee A. Screening for sarcopenia in a small cohort of elderly care home residents using handgrip strength dynamometry; and bioelectrical impedance assessment of skeletal muscle mass and fat free mass. J Aging Res Clin Practice 2012;1(3):219-224.

14. Slee, A, Birch D, Stokoe D. The relationship between malnutrition risk and clinical outcomes in a cohort of frail older hospital patients. Clin Nutr ESPEN. 2016; 15: $57-62$.

15. Landi F, Russo A, Liperoti R, Pahor M, Tosato M, Capoluongo E et al. Midarm muscle circumference, physical performance and mortality: results from the aging and longevity study in the Sirente geographic area (ilSIRENTE study). Clin Nutr 2010;29(4):441-7.

16. Rolfson DB, Majumdar SR, Tsuyuki RT, Tahir A and Rockwood K. Validity and reliability of the Edmonton Frail Scale. Age Ageing 2006; 35(5): 526-9.

17. Collin C, Wade DT, Davies S, Horne V. The Barthel ADL Index: a reliability study. Int Disabil Stud. 1988;10(2):61-63.

18. Kyle UG, Genton L, Karsegard L, Slosman DO, Pichard C. Single prediction equation for bioelectrical impedance analysis in adults aged 20-94. Nutrition 2001;17:248-53.

19. Dupuy C, Lauwers-Cances V, Guyonnet S, et al. Searching for a relevant definition of sarcopenia: Results from the cross-sectional EPIDOS study. Journal of Cachexia, Sarcopenia and Muscle. 2015;6(2):144-154. doi:10.1002/ jcsm.12021.

20. Perna S, Francis MD, Bologna C, et al. Performance of Edmonton frail scale on frailty assessment: Its association with multi-dimensional geriatric conditions assessed with specific screening tools. BMC Geriatrics. 2017;17(1).

21. Villafañe JH, Pirali C, Dughi S, et al. Association between malnutrition and Barthel index in a cohort of hospitalized older adults article information. Journal of Physical Therapy Science. 2016;28(2):607-612.

22. Serrano-Urrea R, García-Meseguer MJ: Relationships between nutritional screening and functional impairment in institutionalized Spanish older people. Maturitas, 2014, 78: 323-328.

23. Zuliani G, Romagnoni F, Volpato S, et al. Nutritional parameters, body composition, and progression of disability in older disabled residents living in nursing homes. The Journals of Gerontology Series A: Biological Sciences and Medical Sciences. 2001;56(4):M212-M216.

24. Cereda E, Valzolgher L, Pedrolli C. Mini nutritional assessment is a good predictor of functional status in institutionalised elderly at risk of malnutrition. Clinical Nutrition. 2008;27(5):700-705. 